\title{
Rapid detection of vaginitis in reproductive age group-by immunochromatography method
}

\author{
Noor Jahan ${ }^{1}$, Tabassum Ghani ${ }^{1}$, Afrina Begum ${ }^{1}$, Taufiqua Hussain ${ }^{1}$, Sahada Anwar ${ }^{2}$ \\ ${ }^{1}$ Department of Obstetrics and Gynaecology, Dhaka Medical College, ${ }^{2}$ Department of Microbiology and Immunology, \\ Bangabandhu Sheikh Mujib Medical University.
}

\begin{abstract}
Vaginitis is the most frequent gynaecologic diagnosis encountered by physician providing primary care to the women. It is defined as inflammation and/or irritation of the vagina, a troublesome condition that affects millions of women in all parts of the world. A total of 50 women of reproductive age within 15-45 years, both pregnant and non-pregnant with abnormal vaginal discharge were enrolled in the study. Vaginal fluid was collected from the patients attending outpatient department of Dhaka Medical College \& Hospital. A rapid vaginal immunochromatography kit were used to test the samples for Chlamydia, Trichomonas, Candida and Gardnerellavaginalis along with $\mathrm{pH}$, nitrites, protein and leucocytes. Bacterial vaginitis was diagnosed in $16 \%$, $20 \%$ and $18 \%$ of the cases using microscopy, culture and rapid vaginal test kit respectively, whereas, was detected in $16 \%, 8 \%, 16 \%$ by microscopy, culture and rapid vaginal kit respectively in case of bacterial vaginosis. Chlamydia(6.66\%) and Trichomonas (6.66\%) was detected in the vaginal kit and Candida was detected in $3.33 \%$ cases using all three methods. Rapid vaginal kit is a simple, rapid test (result within 10 minutes) and can be used as a point of care test or for screening large number of samples.
\end{abstract}

\section{Introduction:}

Vaginitis is defined as a spectrum of conditions that cause vaginal and sometimes vulvar symptoms, such as itching, burning, irritation, odour, and vaginal discharge. It can occur in females of any age accompanied with or without discharge ${ }^{1}$. Vagina represent an ecological niche inhabited by numerous of aerobes and anaerobes coexist in a dynamic balance. It dynamically changed in structure and composition according to age, timing of menstrual cycle, infections, pregnancy, sexual status and personal hygiene. Vulvovaginal complaints are one of the most common reasons for women to seek medical advice ${ }^{2}$.

The most common infectious causes of vaginitis are bacterial vaginosis, vulvovaginal candidiasis, and trichomoniasis. Physicians traditionally diagnose vaginitis using the

\section{Correspondence:}

Dr. Noor Jahan

Assistant Professor

Department of Obstetrics and Gynaecology

Dhaka Medical College, Dhaka

Mobile: 01817-099597 combination of symptoms, physical examination, $\mathrm{pH}$ of vaginal fluid, microscopy, and the whiff test. Vaginitis is frequently asymptomatic or multifactorial. About 90 percent of the vaginitis cases are secondary to vaginosis, valvovaginal candidiasis and trichomoniasis and also chlamydia infection ${ }^{3,4}$. Common symptoms of vaginitis includes itching, burning, pain inside the vagina,skin of the vulva and abnormal or increased vaginal discharge, discomfort and pain during urination and intercourse etc ${ }^{5}$.

This common form of vaginitis can usually be treated effectively if correctly diagnosed. But if left untreated, misdiagnosed or incorrectly diagnosed, it can produce serious consequences like infertility, ectopic pregnancy and eventually lead to major surgery, hysterectomy with bilateral sulphingo oophorectomy ${ }^{6}$.

The prevalence is higher in United States of America (38\%), where as in India the rate of detection is lower $22.65 \%$ (5) Rate of isolation was $22.65 \%$ in Bangladesh ${ }^{7}$. As a multicauses infection, it is imperative for the Gynaecologist to know the exact causative agent to guide a proper treatment ${ }^{8}$. The correct conventional diagnosis is vaginal swab for culture for bacteria and yeast and microscopic examination 
for Trichomoniasis. Earlier method of BV diagnosis relied on Amsel Clinical Criteria which considers factors like raised vaginal $\mathrm{pH}$, positive amine odour, homogenous abnormal vaginal discharge and presence of clue cell on wet film ${ }^{8}$. The present laboratory diagnosis of bacterial vaginosis is based on Nugent scoring system, New proline aminopeptidase assay and BV blue test only can detect bacterial vaginosis. These two tests cannot diagnose the coexistence of Trichomonas and Candida infection al ong with bacterial vaginosis ${ }^{9}$. These methods are not immediately available in many clinical settings, are individually time-consuming, and do not provide timely results.

Despite technological advances in point-of-care testing options, expert recommendations continue to describe a standard of clinical diagnosis via history and physical examination, pH determination, amine (whiff) test, and wet prep microscopy for these common women's health complaints partially due to concerns about increased costs associated with newer options (10). In a review of studies published between 1966 and 2003, the diagnosis of bacterial vaginosis (BV) was made in 22 to $50 \%$ of symptomatic women, candidiasis vaginitis (CV) in 17 to $39 \%$, and Trichomonas vaginalis (TV) in 4 to $35 \%$. Approximately $30 \%$ of symptomatic women remained undiagnosed after clinical evaluation4.

The FemLab ${ }^{\circledR}$ (GIMA, USA) Vaginitis test kit is a screening device for use in the detection of the major forms of vaginitis in vaginal fluid specimens from women concerned about their vaginal health. In a study in USA, out of 106 patients tested, the sensitivity and specificity for a rapid kit was satisfactory $(32-86 \% \text { vs } 91-99 \%)^{11}$. Whereas, this kit showed sensitivity of that was in the agreement with the control methods for the positive diagnosis of the four various disease states - ranging between $84.7 \%$ and $92.0 \%$ and specificity between $78.6 \%$ and $92.0 \%$.

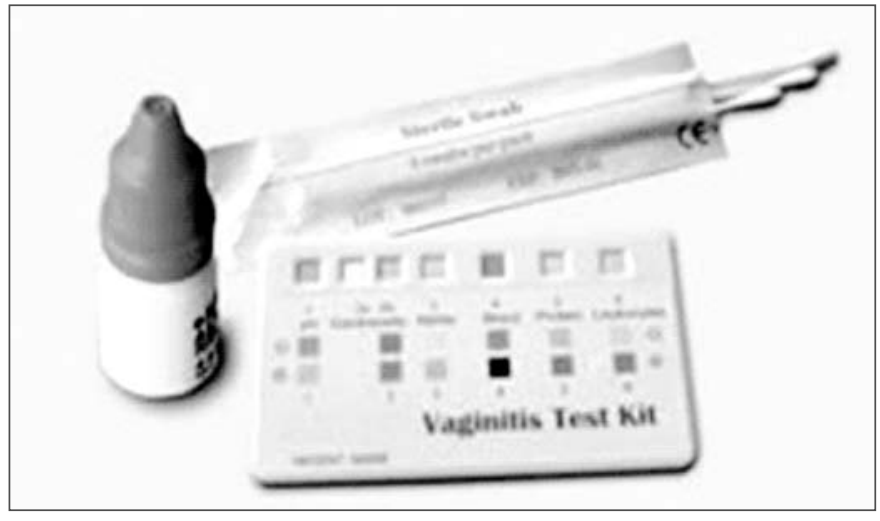

Figurel: FemLab ${ }^{\circledR}$ (GIMA, USA) Vaginitis Test kit
The FemLab Pro test kit has a total of seven sample application zones on the small plastic test platform Zones are for PH (zone 1), Gardnerella zone (zone 2A, 2B), nitrite zone (zone 3), blood zone (zone 4), protein zone (zone 5), leucocyte zone (zone 6). Each test zone and the color changes for each positive and negative result are interpreted according to the kit instruction. The seven test zones are individual chemical and biological tests that screen for specific chemical or biological aspects of the vaginal fluid samples. These test zones can accurately differentiate between the various di sease states.

The purpose of this study was to explore the role of this vaginal kit in the diagnosis of bacterial vaginitis and vaginosis rapidly which might act as a point of care test and guide the clinician to rapid diagnosis and prompt treatment for these patients.

\section{M ethods and M aterials:}

This was a prospective study in which standardized clinical examinations were conducted for women presenting with vulvovaginal symptoms. Vaginal fluid from fifty females of reproductive age, 15-45 years of age, attending out patient department of $\mathrm{DMCH}$ were recruited to participate in the study. Both pregnant and non-pregnant femal es with abnomal vaginal discharge with or without valval itching or burning wereincluded in the study.

After informed consent, participants were interviewed and examined by the clinicians, according to a standard clinical protocol for the diagnosis and management of vulvovaginal symptoms. Women were excluded from participation if they were menstruating or had coitus within the last 24 hours, history of taking antimicrobial agents or vaginal medication within last one month or history of vaginal douche on the day of examination and patient having purulent cervical mucus plug on specul um examination.

Patients were interviewed according to a predesigned data sheet, where a standard clinical history including chief complaint, current symptoms, and self-care for symptoms were noted along with behavioural risk factors, previous history of treatment for genitourinary infection. The clinical examination included a speculum exam for observation of mucopurulent cervicitis and for colour, viscosity, and homogeneity were eval uated.

With all the aseptic precautions, after inserting vaginal specul um into the vagina, three vaginal swabs were collected using the sterile swabs provided in the kit. The swabs were 
kept there for 3 to 5 minutes for better absorbance of the vaginal fluid.

The vaginal samples- two vaginal swabs were tested for wet mount microscopy and sent to the Microbiology Laboratory to detect the presence of Trichomonas, Candida. Gram staining were performed to find out Clue cells and to diagnose bacterial vaginosis. Culture for BV was also performed in the Microbiology laboratory. Vaginal swabs were al so tested using the kit FemLab.Vaginal fluid samples were collected in a threestep collection procedure; two samples were used directly on test zones, and the third was diluted in a custom designed buffer dilution and delivery system. The test results were determined by observing color changes on each test zone. The colorswere compared to a color chart on the test cassette to draw conclusions as to the results of each test and interpreted according to the kit. It gave the col our changes in 10 minutes making it a rapid test

\section{Results:}

A total of 50 clinically suspected cases of vaginitis aged between 15-45 years were recruited in the study. Among them 30 were non-pregnant and 20 cases were pregnant with gestational age ranging from 6 to 32 weeks. Majority of the patients (80\%) were in the age group of 15-35 years. Seventy two percent of the study population were bel ong to lower and middle socio-economic group.

When the vaginal samples were tested by immunochromatography (ICT) kit, among non-pregnant patients, about $33.3 \%$ had bacterial vaginitis followed by bacterial vaginosis (26.7\%). Whereas, in pregnant patients, only $6.66 \%$ had bacterial vaginosis and $6.66 \% \mathrm{BV}$. Fewer of them had Chlamydia, candida and trichomonas, though none of the pregnant patient had trichomoniasis (Tablel).

Bacterial vaginosis was detected $16 \%$ using microscopy but culture yied ded only $08 \%$. Microscopy positive BV was also positive in Rapid vaginal kit detected. The kit could detected more BV than microscopy and culture ( $18 \%$ vs $16 \%$ and $20 \%$ respectively). Chlamydia and Trichomonal vaginitis were undetected in microscopy and culture and were detected by the kit. However, in detecting Candidal vaginitis all three methods showed similar results (Tablell).

Out of 8 bacterial vaginosis cases, diagnosed by microscopy, $6(75 \%)$ were found positive by rapid vaginal kit and only 4 (50\%) were positive by culture. Among the 8 bacterial vaginitis cases, all were microscopy and culture positive, however, with rapid vaginal kit the detection rate was $87.5 \%$.
Table I: Vaginitis detected by the ICT in pregnant and non-pregnant study population.

\begin{tabular}{lccc}
\hline $\begin{array}{c}\text { Clinical diagnosis of } \\
\text { Vaginitis }\end{array}$ & \multicolumn{2}{c}{ No of cases (\%) detected by ICT } & Total \\
\hline & Pregnant & Non-pregnant & \\
Bacterial vaginosis & $08(26.66)$ & $02(6.66)$ & $10(33.3)$ \\
Bacterial vaginitis & $10(33.33)$ & $02(6.66)$ & $12(40)$ \\
Chlamydia vaginitis & $02(6.66)$ & $01(3.33)$ & $03(10)$ \\
Trichomonal vaginitis & $02(6.66)$ & $00(00)$ & $02(6.66)$ \\
Candidal vaginitis & $02(6.66)$ & $01(3.33)$ & $03(10)$ \\
Total & $24(80)$ & $06(20)$ & $30(100)$ \\
\hline
\end{tabular}

Table II: Comparative diagnosis of vaginitis using microscopy, culture and rapid vaginal kit among study population.

\begin{tabular}{lccc}
\hline $\begin{array}{c}\text { Clinical diagnosis } \\
\text { of vaginitis }\end{array}$ & $\begin{array}{c}\text { Microscopy } \\
\text { positive }\end{array}$ & Culture positive & $\begin{array}{c}\text { Rapid vaginal } \\
\text { kit positive }\end{array}$ \\
\hline Bacterial vaginosis & $08(16 \%)$ & $04(08 \%)$ & $08(16 \%)$ \\
Bacterial vaginitis & $08(16 \%)$ & $10(20 \%)$ & $09(18 \%)$ \\
Chlamydial vaginitis & - & - & $03(06 \%)$ \\
Trichomonal vaginitis & - & - & $02(04 \%)$ \\
Candidal vaginitis & $03(06 \%)$ & $03(06 \%)$ & $03(06 \%)$ \\
\hline
\end{tabular}

\section{Discussion:}

Bacterial vaginosis and different forms of vaginitis are themost common presentations in women of reproductive age attending Gynaecology Outpatient Department. It is associated with significant matemal and fetal mortality and following gynaecological operations are now well documented ${ }^{12}$. Its prevalence varies between $5 \%-51 \%$ in different population. This higher prevalence of BV in the obstetric population is held responsible for the higher incidence of preterm labour and complications during pregnancy, which can be prevented by screening and diagnosis of the clinical condition ${ }^{13}$. Early and accurate diagnosis of BV is essential to control BV and to reduce the prevalence. As the clinical signs associated with BV are neither sensitive nor specific, misdiagnosis and delays in treatment can place women at further risk of persistent disease, discomfort, and adverse sequel ae ${ }^{10}$.

The majority of women at the greatest risk of the sequelae of BV are not in settings where conventional diagnostic methods are either practical or possible, and they would greatly benefit from access to rapid and reliable point-of-care tests to improve the diagnosis and management of $\mathrm{BV}{ }^{11}$.

Point-of-care tests have the potential to facilitate prompt diagnosis of BV and provision of the appropriate treatment for BV at the primary visit POC tests that are accurate, simple, rapid, low cost, and stable and that do not require 
high levels of training for their interpretation is integral to improving the management for vaginal discharge. The rapid kit test performed well compared with conventional diagnostic methods for the assessment of women with symptoms suggestive of BV at the bedside and performed better than microscopy and culture methods. Which was also found in other studies ${ }^{14,12}$.

The test was also able to provide a more objective and more rapid diagnosis of $\mathrm{BV}$ at the bedside compared to conventional diagnostic methods. This study needs to be conducted in a population with a high prevalence of BV with a large sample size.

\section{REFERENCES:}

1. Carr PL, Felsenstein D, Friedman RH. Evaluation and Management of Vaginitis. J Gen Intern Med. 1998 May;13(5):335-46.

2. Al-Dahmoshi HOM. Rapid Detection of Microbial Profile among Women with Vaginitis in Hilla City, Iraq. -. J Appl PharmSci. 2017; 7(2):228-32.

3. Martius J, Krohn MA, Hillier SL, Stamm WE, Holmes KK, Eschenbach DA. Relationships of vaginal Lactobacillus species, cervical Chlamydia trachomatis, and bacterial vaginosis to preterm birth. Obstet Gynecol. 1988J an; 71(1):89-95.

4. Rao PS, Devi S, Shriyan A, Rajaram M, J agdishchandra $K$. Diagnosis of bacterial vaginosis in a rural setup: comparison of clinical algorithm, smear scoring and culture by semiquantitative technique. Indian J Med Microbiol. 2004 Mar;22(1):47-50.

5. Bhujwala RA, Buckshee K, Shriniwas null. Gardnerella vaginalis \& associated aerobic bacteria in nonspecific vaginitis. IndianJ Med Res. 1985 Mar;81:251-6.

6. Al-Dahmoshi HOM. Rapid Detection of Microbial Profile among Women with Vaginitis in Hilla City, Iraq. -. J Appl PharmSci. 2017; 7(2):228-32.
7. Akhter $\mathrm{S}$, Satter $\mathrm{H}$, Tarafder $\mathrm{S}$, A min Miah $\mathrm{R}$, Shamin S, Ahmed S. Rapid Detection of Bacterial Vaginosis (BV) by BVBlue test. Bangladesh J Med Microbiol. 2011 Aug 23;4.

8. Davis JD, Connor EE, Clark P, Wilkinson EJ, Duff P. Correlation between cervical cytologic results and Gram stain as diagnostic tests for bacterial vaginosis. Am J Obstet Gynecol. 1997 Sep; 177(3):532-5.

9. Brewer $\mathrm{JI}$, Hal pern $\mathrm{B}$, Thomas $\mathrm{G}$. Hemophilus vaginal is vaginitis. Am J Obstet Gynecol. 1957 Oct;74(4):834842-843.

10. Bradshaw CS, Morton AN, Garland SM, Horvath LB, Kuzevska I, Fairley CK. Evaluation of a Point-of-Care Test, BVBlue, and Clinical and Laboratory Criteria for Diagnosis of Bacterial Vaginosis. J Clin Microbiol. 2005 Mar; 43(3):1304-8.

11. Passos MRL, Varella RQ, Barreto NA, Garcia ML, Giraldo PC. Accuracy of a self-collection kit for the microbiological study of the vaginal content. Braz J Infect Dis. 2007 A pr;11(2):249-53.

12. Easmon CS, Hay PE, Ison CA. Bacterial vaginosis: a diagnostic approach. Genitourin Med. 1992 Apr; 68(2):134-8.

13. McGregor JA, French JI, Parker R, Draper D, Patterson $E$, Jones $W$, et al. Prevention of premature birth by screening and treatment for common genital tract infections: results of a prospective control led eval uation. AmJ Obstet Gynecol. 1995 J ul; 173(1):157-67.

14. Krohn MA, Hillier SL, Eschenbach DA. Comparison of methods for diagnosing bacterial vaginosis among pregnant women. J Clin Microbiol. 1989 J un; 27 (6):1266-71. 